forme également en gelée très pauvre en chaux, que le chlorure de ealeium précipite de nouveau intégralement, sans laisser de traces dans la caséine.

\title{
LES VITAMINES DU LAIT
}

\author{
E. LESNÉ \\ par \\ et \\ H. VAGLIANO \\ Médeein de $\mathrm{l}^{\mathrm{H}} \mathrm{H}$ pital Trousseau. \\ Chef de elinique \\ à la Faculté de médecine d'Athènes.
}

Le problème de la nutrition n'est pas résolu lorsqu'un régime correspond à un nombre de calories suffisant fournies par des substances albuminoïdes (aeides aminés), des graisses, des hydrates de earbone et qu'il renferme une certaine quantité d'eau et de sels. D'autres substances sont indispensables à l'entretien et à la croissance de l'organisme : depuis FUNcK on les appelle pitamines; elles constituent l'une des principales qualités de l'aliment vivant, de l'aliment cru nécessairè à la nutrition. Nous ne savons rien de leur nature chimique; elles n'ont pas été isolées; mais leur présence dans les aliments est démontrée physiologiquement : lorsqu'elles sont présentes, la nutrition est normale, tandis que leur carence produit des phénomènes morbides. Leurs propriétés physiques sont aussi peu connues : les unes sont solubles dans les graisses (vitamine A de Funck, vitamine liposoluble de Me Colnum et Davis); les autres dans l'eau (vitamine B hydrosoluble de Funck, de Me Cosuum), dans l'aleool, l'éther, le ehloroforme. A ees deux vitamines on adjoint la vitamine $\mathrm{C}$ antiseorbutique de DRvммомd. La chaleur les modifie ou les détruit, ainsi que l'oxygène, le cuivre, le radiun.

Le lait, aliment unique du nourrisson, qui suffit à son entretien et à sa croissance, doit done renfermer toutes les vitamines.

STEPp [1] a constaté que si à un régime stérilisé et purifié on ajoute du lait préalablement lavé par l'éther et l'alcool, les souris ne croissaient pas ; les vitamines ont été enlevées par l'éther et l'alcool.

Hopкins [2] soumit de jeunes rats à un régime synthétique composé de protéines purifiées : graisse, amidon, sucre et sels inorganiques; la eroissance et même la vie fut impossible. Si à ce régime on ajoutait 3 ou $4 \%$ de lait eru la croissanee reprenait et la vie se prolongeait à volonté.

Osborne et Mendel $[3,4]$, Mc Colrum et Davis [5], répétèrent et complétèrent avec le même succès les expérienees précédentes. Il faut donner à de jeunes rats soumis à un régime dépourvu de vitamine $\mathrm{B}, 10 \mathrm{cc}$. de lait par jour et par animal pour que la croissance soit normale.

Le lait est done un aliment absolument eomplet, il renferme non seulement les substances nécessaires à l'entretien de la vie, mais, 
destiné à des organismes en voie de développement, il contient de plus les éléments indispensables à la croissance. Le lait renferme les vitamines $A, B, C$.

La glande mammaire fait-elle la synthèse des vitamines, ou bien son rôle consiste-t-il uniquement d̀ éliminer les vitamines contenues dans les aliments ?

Me Collum et Simmonds [6] avaient déjà signalé que les petits rats à la mamelle ne se développaient pas lorsque la nourriture de la mère est privée de vitamines.

Pour élucider cette question dont l'intérêt pratique est évident, nous avons fait une série d'expériences. Avec Wormann [7] nous avons choisi plusieurs femelles de rats sur le point de mettre bas. Ces animaux ont été soumis à un régime avitaminé (riz, easéine, huile d'olives et sels, stérilisés à $120^{\circ}$ ) quelques jours après la naissance des petits. Ceux-ci trois ou quatre jours après le début de l'expérience cessaient de croître. Pour démontrer que cet arrêt de croissance était bien la conséquence du régime avitaminé de la mère, nous avons divisé les petits rats en deux lots: les uns recevaient en plus du lait maternel une petite quantité de vitamines A et B (extraits de levure et de beurre), leur poids reprit aussitôt sa courbe ascendante; les autres exclusivement allaités par la mère mouraient.

Il nous a paru intéressant de reprendre ces expériences sur un animal résistant, le chien, chez lequel la durée de l'allaitement est plus longue [8]. Plusieurs chiennes pleines sont soumises à un régime exclusivement composé de viande et de pain portés à l'ébullition pendant sept heures, régime qui est devenu ainsi très pauvre en vitamine. B. Après plusieurs jours de ce régime, ces chiennes mettent bas plusieurs petits. Les uns sont exclusivement allaités par lá mère tandis que les autres ingèrent ou reçoivent en injection sous-cutanée 10 cc. de vitamine B extraite de la levure de bière. Or, tandis que ceux-ci se développent normalement, les premiers ont une croissance très lente, sont tristes, perdent l'appétit et meurent au bout de dix-neuf jours après avoir présenté de la parésie du train postérieur puis du train antérieur, réalisant un syndrome très voisin du béribéri expérimental tel qu'il a été reproduit par ExkмAN chez les oiseaux nourris au riz décortiqué. Un des jeunes animaux qui était mourant guérit en quelques jours par des injections sous-cutanées d'extrait de levure de bière.

Les chiennes supportaient assez bien la carence en vitamine B, mais leur lait qui, par ailleurs, paraissait de quantité et de qualité normales était avitaminé. Cette expérience se rapproche des faits constatés aux Philippines chez les nourrices atteintes de béribéri, parce que leur nourriture presque exclusive est constituée par du 
riz décortiqué. Leurs nourrissons sont eux-mêmes secondairement atteints de héribéri et guérissent rapidement lorsqu'ils sont confiés à une nourrice saine ou lorsqu'ils sont soumis à l'allaitement artificiel.

Avec Christou nous avons fait les mêmes constatations expérimentales pour la vitamine C [9]. Plusieurs femelles de cobayes ayant mis bas quelques jours auparavant ont été soumises à un régime scorbutigène (foin, son, avoine et lait stérilisé à $120^{\circ}$ ). Comme la femelle de cobaye ne donne de lait que pendant vingt jours environ, les petits ont été nourris successivement par plusieurs femelles soumises au même régime carencé. Or, tous les petits cobayes mouraient de scorbut si l'on ne prenait pas la préeaution de leur donner ou de donner à la femelle les nourrissant, soit par ingestion, soit par injection sous-cutanée une certaine quantité de jus d'oranges.

Les enfants ne paraissent pas devenir seorbutiques, parce que leur nourrice a une alimentation carencée en vitamine C, mais on constate parfois chez eux, dans ces conditions, de l'anémie et des douleurs osseuses, qui peuvent être la conséquence de certaines erreurs de régime de la nourrice.

La glande mammaire n'a donc pas la faculté de faire la synthèse des vitamines $\mathrm{A}, \mathrm{B}, \mathrm{C}$; il faut que ces substances soient apportées à l'organisme maternel par les aliments : le lait ne les renferme qu'à cette condition. La glande mammaire élimine les vitamines comme le rein excrète l'urée ou le chlorure de sodium.

Existe-t-il un rapport entre la quantité de vitamines ingérées par la femelle laitière et la quantité de vitamines éliminées par la glande mammaire?

Incontestablement oui, et toutes les expériences entreprises sur cette question sont concluantes.

Pour préserver du scorbut des cobayes nourrís avec des aliments stérilisés, il faut leur donner du lait eru en quantité variable suivant les différentes époques de l'année, soit $15 \mathrm{cc}$. par jour pendant les mois de mai, juin, juillet alors qu'une ration quotidienne de $50 \mathrm{cc}$. est nécessaire pour les mois d'hiver (Hart, Steenbock, Elis) [10]. Cela dépend exclusivement de l'alimentation de la vache, comme l'ont démontré Hess, Unger et Suplee [11], puis Egkles, Dahle, Mead et Scharer : en employant le lait de la même vache, 40 ec. ont en juin la même valeur antiscorbutique que 60 ec. en janvier. La richesse du lait en vitamine $\mathrm{C}$ est proportionnelle à la quantité de cette vitamine renfermée dans les aliments; les modifications sont parallèles à celles du régime, et apparaissent rapidement. Les mêmes variations de la teneur du lait en vitamines $A$ et B s'observent ausssi dans les mêmes conditions. Des expériences démonstratives ont été réalisées avee du lait de vache sur le rat dès 1918 par STEEN- 
Bock, Boutwell et Kent [12], puis par Kennedy, Dutcher et Eckles, Dahle, ete. $[13,14]$. Les pulpes et les drèches renferment fort peu de vitamine $B$ contrairement aux céréales qui en renferment beaueoup.

John Golding (Cong. intern. de laiterie, oct. 1923) a montré expérimentalement que le régime d'hiver peut réduire la valeur du lait de vache en vitamine A jusqu'au dixième dẹ sa valeur d'été, mais cette valeur peut être rétablie en ajoutant au régime une substance riche en vitamine $\mathrm{A}$ telle que l'huile de foie de morue. D'autre part, en donnant à des jeunes nourrices de l'huile de foie de morue, on augmente la teneur de leur lait en vitamine $\mathrm{A}$; leurs nourrissons ont une meilleure santé et une croissance plus rapide.

La nourriture des femmes allaitant ou des femelles laitières a done une grande influenee sur la composition du lait; la ration alimentaire devra non seulement être suffisante en graisse, hydrates de carbone, protéides et sels, mais encore renfermer des principes vivants crus, riches en vitamines dont la glande mammaire ne peut faire la.synthèse et qui sont des facteurs indispensables à la croissance.

\section{Qualité Des vitamines DU LAIT}

$1^{\circ}$ Vitamine A. - Le beurre du lait est une des principales sources de la vitamine liposoluble, l'expérience l'avait démontré et la science n'a fait que confirmer ce fait. La vitamine $A$, facteur principal de eroissance, existe en abondance dans le lait qui pendant de longs mois est l'aliment exclusif du nourrisson et convient à son développement.

Malgré un préjugé commun, les beurres très colorés ne sont pas de qualité supérieure aux beurres peu colorés. STE en воск a démontré que cette opinion était erronée; la couleur du lait est due à des pigments provenant de l'alimentation de l'animal et est sans rapport avec ses propriétés nutritives. Nous avons préparé un beurre complètement blane et dont la teneur en vitamine A était très supérieure à celle des beurres ordinaires.

La vitamine A a une composition ehimique inconnue ; on Ia distingue par úne de ses caractéristiques physiques et on la nomme vitamine liposoluble. Cependant en malaxant longuement du beurre dans de l'eau, on peut constater dans l'eau de lavage une certaine proportion de vitamine A. Le meilleur procédé d'isolement est la saponification [15]. Nous avons préparé avec H. Wollmaxn [16] un extrait alcoolo-éthéré de beurre qui paraît renfermer une très forte proportion de vitamine A.

La résistance de cette vitamine aux agents physiques est moyenne ; de hautes températures prolongées la détruisent lentement et ineomplètement; la stérilisation du lait aux températures utilisées dans 
l'industrie ne l'attaque pas de faȩon appréciable. Au contraire, une température de $100^{\circ}$ en coopération avec l'oxydation par apport d'oxygène ou tout simplement par un courant d'air détruit très rapidement la vitamine $\mathrm{A}$; aussi bien l'oxydation à la température ordinaire la détruit lentement.

Cette vitamine résiste mal aux rayons ultra-violets.

L'organisme peut utiliser cette vitamine, comme nous l'avons démontré avec M. Wollmann [16], soit par poie entérale, soit par poie parentérale.

20 Vitamine B. - Funk [17], puis Osbonne et Mendel [18], ont obtenu à l'état de pureté, en l'extrayant du lait sec, la vitamine B, régulatrice du système nerveux.

La quantité de vitamine B qui existe dans le lait est assez importante et cette quantité varie avec le régime alimentaire; la glande mammaire l'excrète exclusivement, mais n'en fait pas la synthèse, comme nous l'avons démontré expérimentalement [8]. Elle résiste à la chaleur et pour la détruire il faut de hautes températures $\left(120\right.$ à $\left.130^{\circ}\right)$ ou une ébullition prolongée de sept à huit heures. Mais elle se laisse entrainer par les moindres précipités (SimonNeT), tels que ceux qui sont produits par la stérilisation, et elle résiste mal aux alcalins.

Les rayons ultra-violets n'agissent pas sur cette vitamine. Cette vitamine étant hydrosoluble, doit être recherchée dans la partie aqueuse du lait; la caséine et le lactose en renferment cependant une notable proportion.

Nous avons démontré (Congrès de médecine de Paris, 1922), que la vitamine B passe dans le lait quelle que soit la voie d'introduction dans l'organisme, entérale ou parentérale,

$3^{\circ}$ Vitamine C. - Contrairement aux autres vitamines, celle-ci est peu résistante : la ehaleur, la réaction du milieu, le temps, l'oxydation la détruisent plus ou moins rapidement, aussi avons-nous recherché tout particulièrement les modifications qu'elle peut subir dans les laits employés pour l'alimentation des enfants [19].

La vitamine $\mathrm{C}$, antiscorbutique eomme l'ont démontré les expériences de Holst, FröLICH, ete., est hydrosoluble et se trouve en abondance dans les fruits et les légumes verts. Ingérée avec les végétaux, elle passe ensuite dans le lait.

Elle n'a pu être isolée, aussi sa eonstitution est-elle aussi mystérieuse que celle des autres vitamines. En l'espace de quelques heures elle est détruite par une température de 60 à $70^{\circ}$; elle résiste plus longtemps en milieu acide qu'en milieu alcalin ou même neutre ; c'est peut-être là l'une des raisons de la valeur alimentaire du babeurre acide et des laits fermentés. Elle n'existe ni dans la caséine, ni dans 
le beurre, ni dans le lactose pur, mais seulement dans le petit-lait [20]. A $37^{\circ}$ ou même au-dessous de cette température, la vitamine C perd son activité en dix à vingt jours.

Une ébullition prolongée au delà de cinq à dix minutes est très nuisible à son intégrité, et si la température dépasse $100^{\circ}$ sa destruction est très rapide. En présence de l'oxygène ou même de l'air elle se détruit lentement; l'eau oxygénée ajoutée au lait le prive de sa vitamine. Enfin, d'après Hess et WeInstok des traces infimes de cuivre ou d'autres métaux amènent la destruction catalytique de cette vitamine.

Le temps a une influence nocive constante surtout lorsque cette vitamine se trouve en milieu liquide et exposée à la lumière. Aussi sommes-nous tout à fait de l'avis du professeur MARFAN : il y aurait grand avantage à noter sur les flacons de lait stérilisé la date de la fabrication.

Deux facteurs ont done une influence sur la teneur du lait en vitamine C : la température de stérilisation et la durée de conservation, et bien souvent dans une même préparation ces deux facteurs se superposent pour les earencer en vitamine.

Nous avons étudié à ce double point de vue les différents laits stérilisés employés pour l'alimentation des nourrissons [19].

Les lails stérilisés industriellement à $110^{\circ}$, de même que les laits fixés ou homogénéisés sont scorbutigènes pour les enfants qui en sont nourris exclusivement; ils ne préservent pas du scorbut les cobayes soumis à une alimentation stérilisée. D'après E. Rousseau, le lait homogénéisé plus particulièrement scorbutigène, détient cette propriété de son mode de préparation, qui comprend l'action combinée de la chaleur et de la haute pression avec détente brutale, autant de facteurs qui renforcent l'action -destructive de l'oxygène même à froid. Pour remédier à cet inconvénient, il faudrait, au cours de ces opérations successives, soustraire constamment le liquide au contact de l'air.

Le lait bouilli cinq à dix minutes et le lait stérilisé à domicile, dans un appareil genre Soxhlet, ne sont pas scorbutigènes pour le eobaye : aussi bien le scorbut est exceptionnel chez les enfants nourris avec ces laits.

Le lait condensé sucré préparé à une température qui ne dépasse pas $80^{\circ}$ paraît aussi bien toléré par les cobayes que les laits crus ou bouillis peu de temps. LASSABLIÈre avait du reste signalé que le lait condensé sucré avait conservé intacte sa vitamine $\mathrm{C}$. Au dire de tous les pédiatres les eas de scorbut infantile observés à la suite de l'emploi même exclusif de ce lait, sont tout à fait exceptionnels. Le lait condensé, comme nous l'avons démontré expérimentalement, conserve sa vitamine antiscorbutique pendant plus de quinze mois; 
passé cette date, ses qualités s'atténuent et, après deux ans de fabrication, il est scorbutigène [21]. Il n'est pas d'autre préparation lactée restant inchangée pendant un temps aussi long. Il y a à cela deux raisons : ce lait est stérilisé à basse température et il est conservé dans des boîtes métalliques à l'abri de l'air et de la lumière. Le milieu sucré ne paraît pas être plus particulièrement propice à la conservation de la vitamine $\mathrm{C}$, et en effet nous avons remarqué que l'addition de sucre aux diverses préparations de lait ne modifie en rien leurs propriétés scorbutigènes, à la condition que ce suere soit pur.

Le lait pasteurisé, chauffé pendant un certain temps entre 60 et $80^{\circ}$ a conservé expérimentalement son pouvoir antiscorbutique et la courbe des cobayes nourris avec ce lait se rapproche beaucoup de celle des cobayes nourris au lait cru. Le lait pasteurisé industriellement n'est cependant pas à conseiller en pratique, car la stérilisation est incomplète; il faudrait le consommer très rapidement ou bien le stériliser à nouveau à domicile. Or, une seconde opération de chauffage détruit la vitamine antiscorbutique. Le lait consommé dans les grandes villes est bien souvent pasteurisé dans les fermes productrices ; si ce lait est ultérieurement porté à l'ébullition, il peut devenir scorbutigène, et ainsi peuvent s'expliquer les cas de scorbut infantile observés après emploi d'un lait qui avait été seulement bouilli à domicile.

Le lait sec ou poudre de lait dont la préparation comporte un chauffage très court à $110^{\circ}$, rend de grands services dans l'alimentation de certains nourrissons qui digèrent mal d'autres laits. Si le lait sec a des avantages, il ne présente pas toutes les qualités du lait frais : nous ne pouvons souscrire à l'opinion de certains médecins américains qui soutiennent que le lait sec n'est pas scorbutigène et qu'il est même capable de guérir le scorbut. Si les cas de scorbut infantile sont exceptionnels après l'emploì de lait sec; cela tient à ce que l'usage de cet aliment est récent en France et que le régime des nourrissons n'était pas exclusif ; on y ajoutait du jus de fruits. Les différents laits secs que nous avons expérimentés ne préservent pas du scorbut le cobave soumis à un régime carencé. C'est done un lait avitaminé. D'après HART, STEEвоск, la teneur en vitamine d'une poudre de lait dépend du procédé de fabrication; le procédé Just (procédé du tambour) se montre moins destructif pour la vitamine C que le procédé du spray (Merrell-Soule).

Les cas de scorbut infantile sont rares si l'on songe au grand nombre d'enfants nourris au lait stérilisé. L'avitaminose n'est pas la cause exclusive de cette affection et la question de terrain est très importante : l'organisme semble pouvoir s'adapter à la carence partielle du facteur antiscorbutique. Il est des résistances indivi- 
duelles surprenantes : tel le cas signalé par Bracri et CARA eoncernant deux jumeaux nourris au même lait stérilisé; l'un d'eux seulement contracta le scorbut.

Vitamine antirachitioue. - Pappenheimer et ses collaborateurs ont démontré que le beurre était dépourvu de facteur antirachitique; donné à des rats soumis à un régime rachitigène il ne les préserve ni ne les guérit du rachitisme que l'on détermine facilement chez eux, opinion contraire à celle admise antérieurement par Mellamby, Me Callum, ete. Nous avons confirmé les expériences de Pappenhermer et démontré également que le lait de femme n'était pas antirachitique [22]; si les enfants élevés au sein ne deviennent pas en général rachitiques, ce n'est donc pas parce qu'ils absorbent un facteur antirachitique contenu dans le lait.

On sait depuis longtemps déjà que l'huile de foie de morue ingérée agit de façon très efficace sur le rachitisme infantile et sur le rachitisme expérimental [23]. Bien que cette action soit tout à fait indépendante de celle de la vitamine A liposoluble [24], nous nous sommes demandé si les propriétés antirachitiques de l'huile de foie de morue ingérée ne pourraient pas être transmises par le lait après exerétion par la glande mammaire, à l'exemple des vitamines A, B et C. L'expérimentation a confirmé cette hypothèse : en ajoutant à l'alimentation normale d'une vache laitière une dose quotidienne de $500 \mathrm{gr}$. d'huile de foie de morue (cette forte dose est nécessaire pour obtenir des résultats, et est fort bien tolérée), on obtient un lait doué de propriétés particulières [25].

Ce lait ne présente aucune modification de couleur, d'odeur ou de saveur.

Le beurre est moins coloré, mais reste aussi abondant; le lait est très riche en lécithine ( $1 \mathrm{gr}$. 40 par litre, dosé en $\mathrm{P}^{2} \mathrm{O}^{5}$ ).

Expérimentalement ce beurre est très riche en vitamine de cròissance liposoluble comme nous l'avons démontré expérimentalement sur des jeunes rats [25]. DALYEL [26] avait fait la mème constatation sur des nourrissons allaités par des femmes qui absorbaient de l'huile de foie de mórue ; l'augmentation de poids de ces nourrissons était alors beaucoup plus rapide que dans les périodes où le régime des nourrices était normal.

Le beurre des vaches qui ingéraient de l'huile de foie de morue s'est montré doué de propriétés préservatives et curatives des plus nettes lorsque nous l'avons employé chez de jeunes rats soumis au régime rachitigène de Pappenheimer.

Donné à des enfants dōnt le rachitisme était en évolution, le lait a agi d'une façon très efficace sur l'état général et sur les lésions osseuses, moins rapidement cependant que si l'on fait ingérer I'huile de foie de morue directement aux petits malades. 
L'organisme des jeunes rats n'accumule pas le facteur antirachitique. En effet, comme l'ont montré Hess et Wernstock [27], l'ingestion d'huile de foie de morue par des femelles en état de gestation ou de lactation ne préserve pas du rachitisme les petits soumis ultérieurement à un régime rachitigène.

En résumé, le lait d'une vache traitée par l'huile de foie de morue à hautes doses, fournit un beurre très riche en léeithine et en vitamines de croissance : l'huile de foie de morue mérite done d'être recommandée aux nourrices.

Ce beurre renferme un facteur antirachitique différent de la vitamine $\mathrm{A}$, ear son extrait reste inefficace par voie parentérale. II agit par ingestion de façon préventive et curative sur le rachitisme expérimental du rat et paraît doué d'une certaine valeur thérapeutique dans le rachitisme infantile en évolution.

\section{Conclusions}

La qualité d'un lait ne peut être déterminée exelusivement par une analyse physicochimique.

Le lait est un aliment vivant, il renferme les vitamines liposoluble et hydrosoluble nécessaires à la croissance et à l'entretien du . nourrisson et la vitamine antiscorbutique.

La nourrice ou la femelle laitière sont incapables de réaliser la synthèse des vitamines; le lait n'en contient que dans la proportion où elles existent dans l'alimentation. Le régime de la nourrice doit done être varié et, de plus, renfermer des crudités riches en vitamines $\mathrm{C}$; il faut pour la même raison surveiller l'alimentation des femelles laitières.

Les vitamines ou, au moins certaines d'entre elles (vitamine $\mathrm{C}$ antiscorbutique), sont fragiles et modifiées ou détruites par la chaleur, la lumière, l'air ou le vieillissement.

La production d'un lait eru aseptique est irréalisable. Cependant la crainte de l'avitaminose ne doit pas faire écarter la stérilisation du lait qui constitue le plus grand progrès de l'hygiène du premier âge pour les enfants qu'on est malheureusement obligé d'allaiter artificiellement.

Les laits les moins carencés en vitamines sont : le lait bouilli cinq à dix minutes, le lait stérilisé à domicile et le lait condensé sucré (conservé en boîtes métalliques à l'abri de l'air et de la lumière).

Les autres préparations lactées (lait sec, lait homogénéisé, etc.), tout en étant scorbutigènes correspondent à certaines indications diététiques et peuvent rendre de grands services dans l'allaitement artificiel. Il sera toujours facile de rendre ces laits inoffensifs en donnant simultanément aux nourrissons du jus de eitron ou d'orange. Aussi bien comme le lait est très pauvre en vitamine antiscorbutique, 
cette mesure préventive nous paraît utile à généraliser dès l'âge de quatre ou cinq mois chez tous les enfants allaités artificiellement.

[1] Stepp, J. Biol,, t. LVII, p. 135, 1912.

[2] Hopkins, Journ. Physiol., t. XLIV, p. 425, 1912.

[3] Osborne et Mendex. Publ. Carn. Inst. Wash, no 156, part. I et II.

[4] Osborne et Mendel, Journ. Biol. Chem., t. XIII, p. 233, 1912.

[5] Me. Collum et Davis, Journ. Biol. Chem., t. XV, p. 67, 1913.

[5] Mc. Colnum et Srmmonds, Am. Journ. Physiol., t. XLVI, p. 275, 1918.

[7] Wollmann et Vagliano, C. R. Acad. des Sc., p. 1637, 19 juin 1922.

[6] Lesné et Vagliano, C. R, XVI e Congrès français de Médecine, p. 146, Paris, 1922.

[9] Lesné, Vagliano et Christou, C. R. Acad. des Sc., p. 1006, 9 avril 1923.

[10] E.-B. Hart, H. Stennbock et W.-R. Ellis, Journ. Biol. Chem., t. XLII, p. $383,1920$.

[11] Hess, Unger et Supplee, Journ. Biol. Chem., t. XLV, p. 229, 1920-21.

[12] Steenbock, Boutwell et Hazel-E. Kent, Journ. Biol. Chem., t. XXXV, p. 517, 1918.

[13] Kennedy et Dutcher, Journ. Biol. Chem., t. L, p. 339, 1922.

[14] A. Dutgher, Ecrles, Dahie, etc., Journ. Biol. Chem., t. XLV, p. 119, 1920-1921.

[15] J.-F.-M. Glendon, Journ. Biol. Chem., t. XLVII, p. 411, 1921.

[16] E. Wollmann et Vagliano, C. R. Soc. Biol., p. 336, 10 février 1923,

[17] Funk, Journ. Physiol., t. XLV, p. 75, 1912 et Journ. Biol. Chem.. t. Ier, p. 27, 1916.

[18] Osborne et Mendel, J. Biol. Chem., t. XXXIV, p. 537, 1918 et t. XI,I, p. 515, 1920.

[19] Lesné et Vagliano, Le Nourrisson, novembre 1922.

[20] Lesné et Mule Dubreuin, Bull. de la Soc. de Pédiatrie de Paris, p. 29, 1923.

[21] Lesné et Vagliano, C. R. Soc. Biol., 16 février 1924.

[22] Lesné et Vagliano, C. R. Soc. Biol, t. XCI, p. 143, 1924:

[23] Schaвad, Zeitsch. f. Klin. Med., t. XVIII, p. 94, 1909 et t. LXIX, p. $435,1910$.

Park et Howland, Bul. of the Johns Hopk. Hosp., t, XXXIII, p. 391, 1921.

Lesné, Vagliano et Christou, Soc. de Pathol. comp., 10 juillet 1923.

[24] Lesné et Vagliano, C. R. Acad. des Sc.; octobre 1923.

[25] Lesné et Vagriano, C. R. Acad. des Sc., t. CLXXIX, p. 539, 15 septembre 1924.

[26] Dalyel, Brit. Méd. Journ., juillet 1920.

[27] Hess et Wernstock, Am. J. of dis. of children, t. XXVII, p. 15, janvier 1924. 